\title{
Carbonification, Carbonization and Graphitization as Studied by High Resolution Electron Microscopy
}

\author{
by A. Oberlin, G. Terriere and J. L. Boulmier, (CNRS ER. 131), \\ Laboratoire Marcel Mathieu, Université d'Orléans,
} 45045 Orléans-La-Source, FRANCE

\section{INTRODUCTION}

When organic material is heat-treated in an inert atmosphere, with a predetermined heating program, from ordinary temperature to $3000{ }^{\circ} \mathrm{C}$, different stages are usually distinguished. The first one occurs either through a solid phase or through a plastic phase and always gives a solid product. During this stage volatile matter is given off. In a second stage, mostly gases are evolved. In the same way, during heat-treatment, the carbon content of the material gradually increases until it reaches $100 \%$ at the end of carbonization. Numerous properties, as ESR, resolidification, resistivity etc.. show some "accident" in their variation, but, unfortunately, it has been impossible up to now to correlate this accident to the temperature $\mathrm{T}$ or to the carbon content $\mathrm{C}$ limiting the two stages of carbonization ( $\mathrm{T}$ varies from $500^{\circ} \mathrm{C}$ to $700^{\circ} \mathrm{C}$ and $\mathrm{C}$ varies from 90 to $94 \%$ of carbon, depending on the material studied and the experimental conditions chosen). Till now the upper limit of the carbonization process cannot be defined either, because the gases produced $\left(\mathrm{H}_{2}, \mathrm{CH}_{4}\right)$ do not leave the samples at a definite temperature ${ }^{1,2)}$ and because no abrupt change may be recognized in the properties. The pure carbon occurring at the end of carbonization, when heat-treated, may be able or not to graphitize progressively (soft or hard carbon).

In nature we can find series of carbonaceous materials belonging to any of the stages defined above (carbonification). For carbon contents lower than $\mathrm{C}$, we have for instance kerogens and coals. Between $\mathrm{C}$ and pure carbon we find anthracites and metaanthracites. Their formation is generally accompanied not only by burial but essentially by tectonic stresses, i.e. shear stresses. These materials, when heat-treated, cannot be divided into two classes (hard or soft carbons) as the industrial carbons. Anthracites look exactly like hard carbon up to $2000^{\circ} \mathrm{C}$, are inhomogeneous between $2000^{\circ} \mathrm{C}$ and $2500^{\circ} \mathrm{C}$ and become graphitizable as soft carbons above $2500^{\circ} \mathrm{C}^{3}$. At the top, we have graphite.

Not many things are known either on carbonization or on carbonification. X-ray workers know that in the patterns some diffuse peaks appear, close to the position of the prominent peaks of graphite, which suggest the existence of stacks of aromatic layers. Some of the authors assumed the existence of some kind of amorphous carbon, attached as peripheral groups to the graphite nuclei ${ }^{4,5)}$ Unfortunately most workers failed to give coherent results, even for the more advanced stage of graphitization ${ }^{6}$. The principal reasons of the difficulties encountered in X-ray diffraction studies are the following ${ }^{7,8,9)}$ :

1) Elementary domains (crystallites) are very small and give only Debye-Scherrer patterns, the rings of which are more and more faint and diffuse as carbon content is lower;

2) Even for high carbon content, the structure is turbostratic and $(h k l)$ rings are replaced by asymmetrical ( $h k$ ) diffuse bands.

All these inconveniences and others, lead to statistical effects, which prevent any individual description of a given crystallite. For instance, it is impossible to distinguish between variations in the interplanar distance between two adjoining carbon layers, or distortion of each layer leading to a fluctuation of the atomic level ${ }^{10}$. All these disadvantages are responsible for a lot of misunderstandings and for a lack of coherent and homogeneous theory of carbonization and graphitization. In spite of that, it is necessary to acknowledge the fact that carbonization, as well as graphitization, cannot be described without taking into account the progressive association of more and more neighbouring elementary domains. This fact is the only condition for crystal growth to occur. It is the reason for which most X-ray workers have been obliged to start from various hypotheses, concerning mutual relations between crystallites.

It is very important to predict the ability of a given carbon to graphitize and consequently, we must answer the question, why are some carbons able to graphitize while some others are not? In other words, what is the graphitization mechanism? It should be equally important to correlate natural transformations to laboratory experiments. We think those questions may be partially answered by the work we have done in using very high resolution electron microscopy. 


\section{ELECTRON MICROSCOPE TECHNIQUES}

The main advantage of the electron microscope as against X-ray diffraction, is that the objective lens of the microscope automatically performs Fourier synthesis of the object (instead of calculation which normally starts from X-ray patterns).

\subsection{Bright-field electron microscopy}

If we consider first the Abbe-image A situated in the back focal plane of the objective lens (fig. 1.), we can see that it corresponds to the diffraction figure of the object (points where each diffracted beam is brought into focus). Because of the smallness of the electron wavelength $(0,037 \AA$ for $100 \mathrm{keV})$, this image may be considered, in first approximation, as a plane section of the reciprocal space. It represents the first Fourier transform of the object, i.e. the equivalent of an X-ray diffraction pattern (non-localized fringes). Each point of the optical image $G$ shows a perfect correspondence with each point of the object (see fig. 1). This means that we obtain, in the Gaussian plane, a second Fourier transform which again gives us the real space. If the lens is perfect the objective takes into account the phases of each of the diffracted beams which can interfere with the incident beam, so that we may automatically obtain the atomic structure of the object.

Unfortunately, the lens is not perfect. It is always more convergent for those rays which are oblique in respect to the optical axis, than for the paraxial ones. This aberration $C_{s}$ (spherical aberration) cannot be corrected and causes a phase shift, proportional to $(2 \theta)^{4}$. Any variation in the focal length of the objective lens introduces a phase shift proportional to $(2 \theta)^{2}$. In addition, each diffracted beam has a phase difference of $\frac{\pi}{2}$ with the undeviated beam. So we may write the total phase difference in the Gaussian plane as follows:

$$
\cos \chi=\cos \left(\frac{\pi}{2}-\frac{2 \pi}{\lambda} C_{s} \frac{(2 \theta)^{4}}{4}+\frac{2 \pi}{\lambda} \Delta f \frac{(2 \theta)^{2}}{2}\right)
$$

If all the other lenses of the electron microscope (projector lenses) are focused on the Gaussian plane of the objective lens, we can see on the fluorescent screen an enlarged image of the object and we are in brightfield electron microscopy. The above formula shows that we may compensate $C_{s}$ for a limited number of diffracted beams, and so directly resolve the atomic structure $^{11)}$ if $\cos \chi \simeq \pm 1$. This can be obtained by choosing a suitable value for $\Delta f$. Unfortunately, in the case of carbon samples, the number of diffracted beams able to interfere is too small to use this technique. Therefore, bright-field images are used for morphological studies only.
We may improve the contrast of the images by placing in the Abbe-plane an aperture, small enough to allow only the incident beam to go through. Because of the withdrawal of all the diffracted beams, each region of the object which emits such a beam, will appear darker than its neighbours. We thus will obtain diffuse sinuous dark fringes in the particle (extinction contours or isocline fringes).

\subsection{Selected area electron diffraction (SAD)}

Fig. 1. shows that if we focus the projector lenses on the Abbe image and if we place a small aperture (20 to $80 \mu \mathrm{m})$ in the Gaussian plane $G$, this is equivalent to inserting a smaller aperture in the object plane 0 . If $L$ is the diameter of the aperture and $M_{0}$ the magnification of the objective lens, thus the effective limitation in size of the object is $l=L / M_{0}$. We will observe on the fluorescent screen the diffraction pattern of a small region of the object $(l<1 \mu \mathrm{m})$.

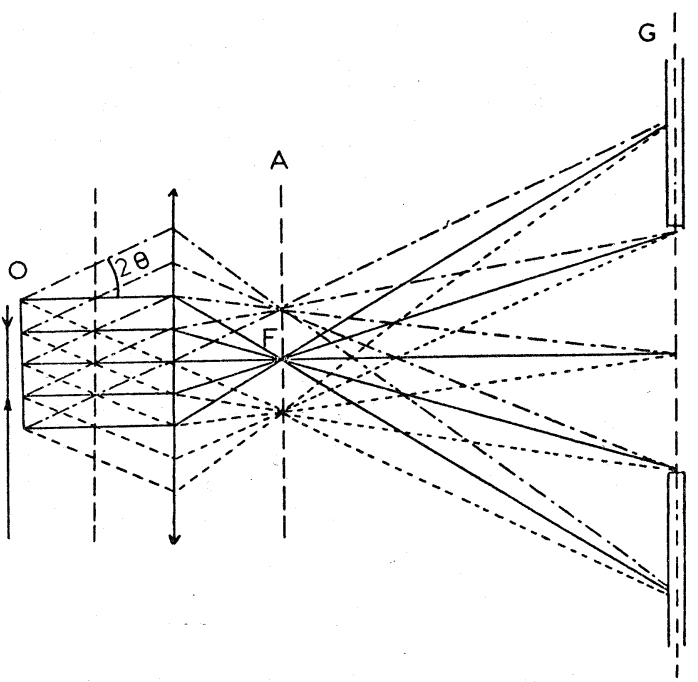

Fig. 1 Ray path in bright-field electron microscopy.

\subsection{Lattice imaging}

Instead of placing an aperture in the Gaussian plane as in fig. 1, and then focusing the projector lenses on the Abbe image, we may place an aperture in the focal plane and focus the projector on the Gaussian plane. If we use an aperture large enough to let through the incident beam and an ( $h k l$ ) diffracted beam (fig. 2), and if we use a suitable defocus of the objective lens, we will obtain in the Gaussian plane a set of dark and bright fringes ${ }^{12)}$ whose spacing $D$ will be $D=M_{0} \cdot d_{h k l}$. In first approximation we will see on the fluorescent screen an enlarged projection of the $(h k l)$ lattice planes onto the supporting film. Owing to the smallness of the 


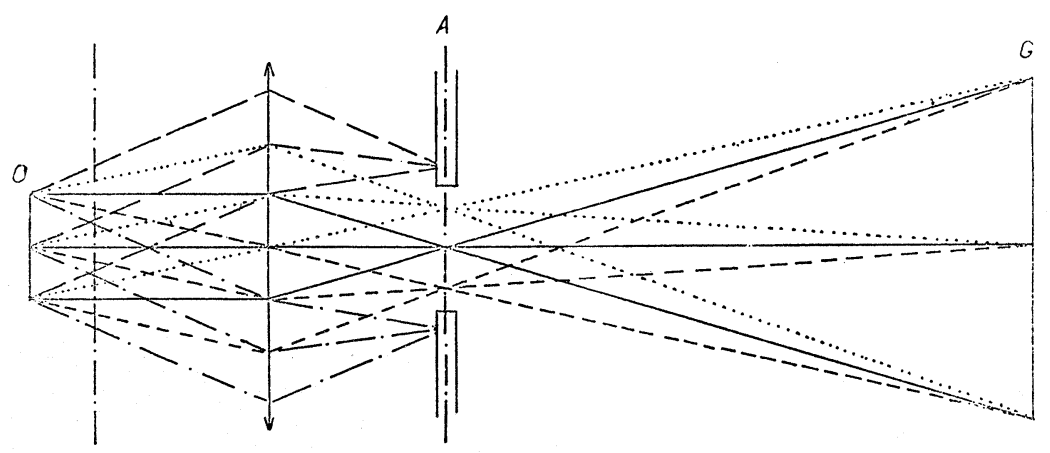

Fig. 2 Lattice imaging.

electron wavelength, we must recall that the Bragg angles are very small and therefore, the $(h k l)$ planes give a diffracted beam only when they are nearly parallel to the incident beam. If we have a fairly good tridimensional crystal, any tilt of the $(h \mathrm{kl})$ planes from the Bragg position will make the $(h k l)$ beam disappear (extinction contours should be very dark and very narrow). The lower the crystallinity will be, the larger the interference error could be, i.e. the deviation of the $(h k l)$ planes from the Bragg position, without disappearance of the diffracted beam, in this case, the intensity of this diffracted beam should be lower (wide, diffuse and faint extinction contours).

For carbon samples, we use the interference between the $(00.2)$ beam and the incident one, so that we are able to see the carbon layers edge on. In order to improve the resolving power and to avoid many possible artefacts (such as the apparition of ghost fringes, with half the normal period), we have tilted the direct beam half-way between the paraxial position and the (00.2) position. $^{13)}$

Because of the extremely high magnification used, lattice imaging shows only a tiny part of the material and consequently it is not reliable if the sample is not homogeneous. Another difficulty arises from the fact that the lattice fringes represent only the projection of the lattice planes, therefore the carbon layers should appear as dark fringes whatever their third dimension may be, i.e. whatever there shape may be (isometric or ribbon-like). We must add that if some graphite-like layers are not grouped at least in pairs, they cannot be seen by this technique. Finally, we should also recall that the directly resolved lattice image is, in fact, a very restricted Fourier synthesis of the crystal, which only reveals certain coarse features of the structure. For instance, lateral shift of the fringes, as well as changes in their spacings, both depend on the thickness or the orientation of the sample and on the focusing of the objective lens. This has led us to use at least one or two other techniques together with lattice imaging. For instance, we must correlate the results obtained with those deduced from dark-field technique.

\subsection{Dark-field electron microscopy}

We may place again an aperture in the Abbe-image (fig. 3), but we choose it small enough to allow only a small part of a given $(h \mathrm{kl})$ beam to go through. If we do so, and use this part to form the optical image (the projector lenses remaining focused on the Gaussian plane) we will obtain a bright image upon a dark-field. In fact, the displacement of the aperture in the Abbe plane is replaced by a tilt of the incident beam with the aperture remaining paraxial. This is strictly equivalent and has the advantage of avoiding spherical aberration effect (loc. cit. 12). The regions of the object which should appear bright in the dark-field image, are those from which the selected $(h k l)$ beam is issued. Anyway, if the aperture does not intercept any beam, the optical image remains completely dark. If the size of the ordered regions of the object decreases, the diffuseness of the $(h k l)$ beams that they produce increases and the size of the bright regions of the image decreases, but they still remain very brightly lit. Provided that the size

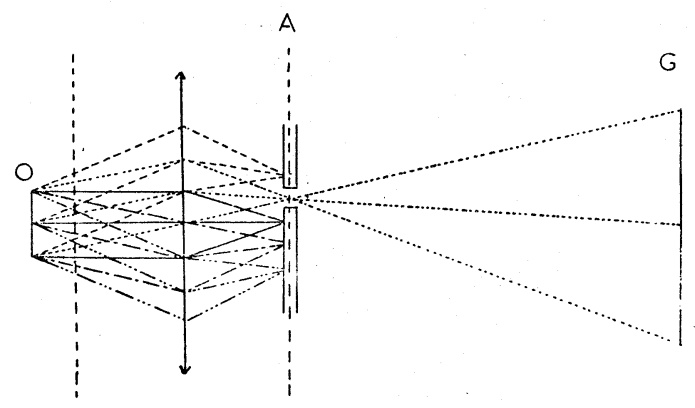

Fig. 3 Ray path in dark-field electron microscopy. 
of the aperture used in the Abbe image is large enough to avoid diffraction effects, we may measure the size of the crystallites for each $(h k l)$ beam ${ }^{14)}$. We have estimated the size of the Airy discs given by the apertures we have used, i.e. half the width of the central diffraction peak given by the aperture in the optical image plane. We have found less than $6 \AA$ for a $10 \mu \mathrm{m}$ diameter aperture (and less than $12 \AA$ for the $5 \mu \mathrm{m}$ one).

Recently ${ }^{15,16)}$ we have modified this dark-field technique in the following manner. We have used apertures of various sizes and we have gradually tilted the incident beam, so as to explore radially the reciprocal space available. For each position of the aperture in respect to the incident beam, we have first taken a picture of the dark-field image. Then, on the next picture, we have

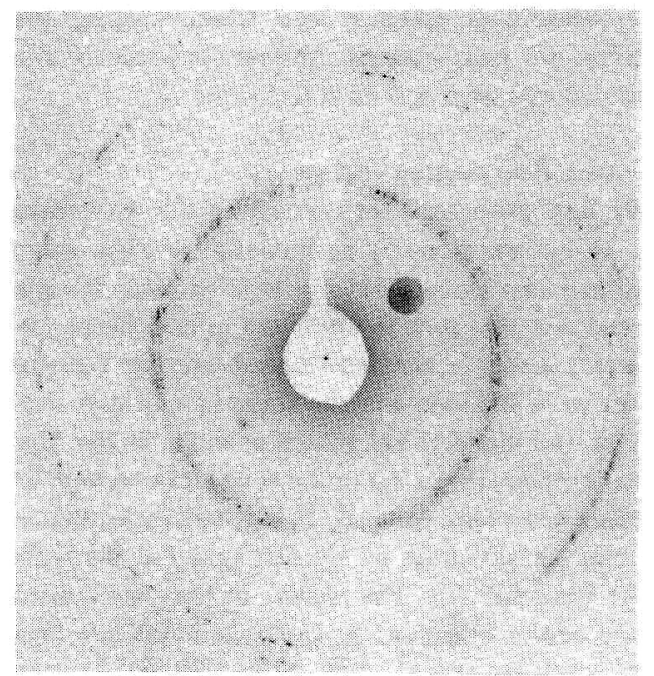

Fig. $45 \mu \mathrm{m}$ objective aperture superimposed upon graphite pattern.

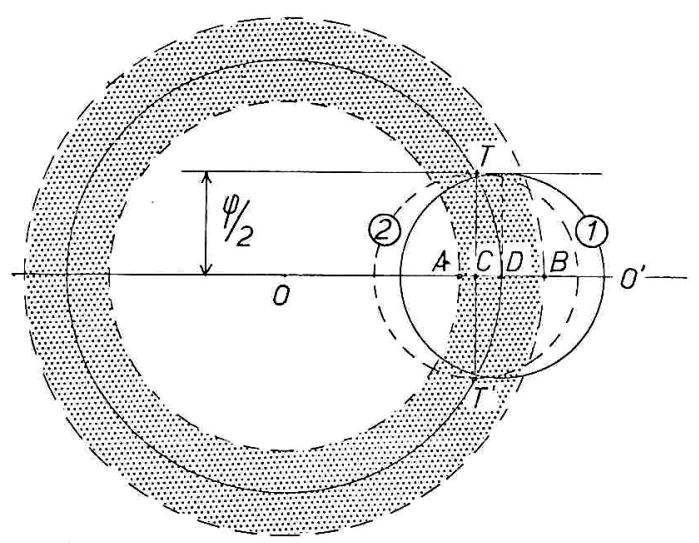

Fig. 5 Interception of an $(h k l)$ ring by the objective aperture. superimposed the aperture contour to the Abbe image (fig. 4). Finally, we have taken a third picture, giving the SAD pattern of a suitable internal standard. We thus know exactly the value of $|\vec{s}| \simeq \frac{2 \theta}{\lambda}$ corresponding to the center of the aperture, from which we may deduce the corresponding value of the $d$ spacing. The whole experimental procedure may be followed by eye on the fluorescent screen.

Fig. 5 shows a Debye-Scherrer ring of a given microcrystalline sample whose radius (which we intend to measure) is $O D=1 / d_{h k l}$, and whose width is represented by the dotted portion of the figure. In this figure we have represented an aperture whose diameter is $\varphi$ and whose center is progressively shifted from the origin toward the high angles. On the figure we may see that, when the aperture is tangent in $A$ to the Debye-Scherrer ring, the crystals giving a diffracted beam centered on $D$ begin to light up. They disappear when the opposite edge of the aperture reaches $B$. These crystals will be the first ones to appear in the dark-field image, and the last ones to disappear. If the width of the DebyeScherrer ring is larger than the aperture or about the same size, the crystals reach their maximum intensity in the optical image only when the center of the aperture is in $D$ in fig. 5 (position number 1 on full line). If the width of the ring is considerably smaller than the aperture diameter, the crystals light up before the aperture center reaches $D$ and after it has been displaced toward $B$. The maximum error thus introduced is equal to the diameter $\varphi$ of the aperture. In the case of the (00.2) ring of graphite, we have found $2,6 \AA<d_{00.2}<4,8 \AA$ for the $10 \mu \mathrm{m}$ aperture and $2,9 \AA<d_{00.2}<3,9 \AA$ for the $5 \mu \mathrm{m}$ aperture, instead of $3,35 \AA$. Therefore we must use the smallest aperture available so as to minimize this error. We will consider now the effect of the radius of curvature of the $(h k l)$ ring. We see in Fig. 5 that the aperture, when centered upon $C$ in position number 2 (dotted line) intercepts all the diffracted beams situated between $T$ and $T^{\prime}$ ( $T$ and $T^{\prime}$ are the intersections between the $(h k l)$ ring and the two lines parallel to 00 , representing the common tangent to the displacing aperture). At first, we can see that, when the aperture is moved, the crystals emitting beams located either in $T$ or in $T^{\prime}$ should light up after those giving beams located between $T$ and $T^{\prime}$. They should reach their maximum intensity immediately and disappear as soon as the aperture center goes beyond $C$. We can also see on the figure that $C D$ represents the error introduced by the curvature of the $(h k l)$ ring in the measurement of $O D$ $=1 / d_{h k l}$ 


$$
C D=\frac{1}{\mathrm{~d}_{h k l}}-\left(\frac{1}{\mathrm{~d}_{h k l}^{2}}-\frac{\varphi^{2}}{4}\right)^{1 / 2}
$$

In order to reduce $C D$ to its minimum, we must again use an aperture as small as possible. For instance, for (00.2) of graphite, we have calculated the value of the error and we have found $\pm 0,05 \AA$ for the $5 \mu \mathrm{m}$ aperture, as against $\pm 0,2 \AA$ for the $10 \mu \mathrm{m}$ aperture. In short, we see that in any case, the smaller the aperture, the smaller the error. Consequently, we have used the $5 \mu \mathrm{m}$ aperture when testing our method experimentally. We have seen that Fig. 4 shows the contour of this aperture superimposed to the (00.2) reflections of a graphite pattern. To obtain this picture, we proceeded as follows: we displaced the aperture toward the high angles in the vicinity of the (00.2) diffracted beam while we examined the dark-field image on the fluorescent screen (near the cross-over illumination in order to obtain the maximum light permissible). We stopped moving the aperture from one direction to another when the bright regions of the graphite particle were lit at their maximum intensity as defined by eye. Then we went back to the Abbe image and took the micrograph. We repeated the same measurements 50 times and we found $3,3 \AA<d_{00.2}<3,5$ $\AA$ instead of $3,35 \AA$. We can see that the experimental spreading of the results is much lower than the maximum value of the above calculated errors $(2,9 \AA$ $3,9 \AA$ ). We shall use this experimental fork for all those materials whose maximum intensity is visible by eye (even for those which have a poor crystalline organization). Naturally, for all samples studied we have used the $5 \mu \mathrm{m}$ aperture only to measure the $d$ spacings and we have used the $10 \mu \mathrm{m}$ aperture to obtain a second set of dark-field micrographs, in order to measure the real size of the carbon layer stacks.

\section{RESULTS}

\subsection{Soft carbons}

We will present herein the results obtained on an anthracene char (sample $n^{\circ} 5$, chosen as a typical soft carbon by the French Carbon Committee). The samples were heat-treated at various temperatures $\left(50^{\circ}\right.$ to $100{ }^{\circ} \mathrm{C}$ intervals) between $400{ }^{\circ} \mathrm{C}$ and $2900{ }^{\circ} \mathrm{C}$.

In a previous work, ${ }^{17,18)}$ we studied carbon samples heat-treated between $1000{ }^{\circ} \mathrm{C}$ and $3000{ }^{\circ} \mathrm{C}$ and we found three stages called A, B, C. The first one extended from $1000{ }^{\circ} \mathrm{C}$ to $1600{ }^{\circ} \mathrm{C}$, the second one from $1600{ }^{\circ} \mathrm{C}$ to $2000^{\circ} \mathrm{C}$ and the third one from $2000^{\circ} \mathrm{C}$ to the end of graphitization process. Actually, the samples heattreated below $1000^{\circ} \mathrm{C}$ are still being studied in the. laboratory. First, we found the occurrence of an impor- tant dispersion in the values of the $d_{00} 2$ spacings, as measured by dark-field techniques. Second, we have: found some variations in the values of the misorientation of two neighbouring stacks of carbon layers. From our data, we have shown that there is no transition between two different stages at $1000{ }^{\circ} \mathrm{C}$ but at a lower temperature which is not yet precisely determined. In the present work, because of these new results, we have changed the stages previously described from A, B, C to III, II, I (with I corresponding to the highest degree of graphitization). For the above reasons we have arbitrarily chosen the sample heat-treated at $1000{ }^{\circ} \mathrm{C}$ as starting sample of stage III.

The degree of graphitization $g$ has been determined from the values of the interlayer spacing $d_{00.2}$ deduced from X-ray diffraction spectras by using $\mathrm{CuK}_{\alpha}$ or $\mathrm{CoK}_{\alpha}$ radiations. In order to obtain sufficient accuracy in $g$ values, it is necessary to determine $d_{00.2}$ within a precision of $0,001 \AA$. We have always used samples containing an internal standard, studied by transmission techniques, to avoid peak displacements due to X-ray absorption effects by the internal standard (a Guinier camera is particularly suitable for this purpose). The standard is chosen in such a way as to produce two peaks between which the $(00.2)$ carbon reflection is located. The $d_{00.2}$ : spacing is thus deduced by interpolation from microphotometric records. For all samples where $3,40 \AA<d_{00.2}<3,45 \AA$ we have used a half-andhalf mixture of quartz $\left(d_{101}=3,343 \AA\right)$ and anatase $\left(d_{101}=3,516 \AA\right)$, whose peak intensities are comparable. For the $d_{00.2}$ spacings smaller than $3,40 \AA$ we have chosen synthetic $\mathrm{BaSO}_{4}$ giving $d_{210}=3,442 \AA$ and $d_{102}$ $=3,317 \AA$. The proportion of the internal standard to be used must be determined experimentally in each case.

\subsubsection{Stage III (below $1600^{\circ} \mathrm{C}$ )}

The bright-field micrographs show that the particles obtained through gentle manual grinding have a lamellar shape and lie flat on the supporting film. ${ }^{19)}$ It is a wellknown fact that there is a strong preferred orientation of the layer planes which tend to be parallel to the plane of the lamellaes. Therefore, in SAD patterns no (00.2) reflection appears, except in the region where the thinnest particles are folded. These-regions are the only ones appearing brightly in the (00.2) dark-field images and they may be used for lattice imaging.

The lattice imaging of the sample heat-treated at $1000{ }^{\circ} \mathrm{C}$ is shown in Fig. 6. In this micrograph, small layer plane segments appear, arranged in parallel stacks whose length $L_{1}$ is below $10 \AA$. They are separated from each other by tilt (and probably twist) boundaries, giving about $\pm 10^{\circ}$ misorientation. The number of layers 


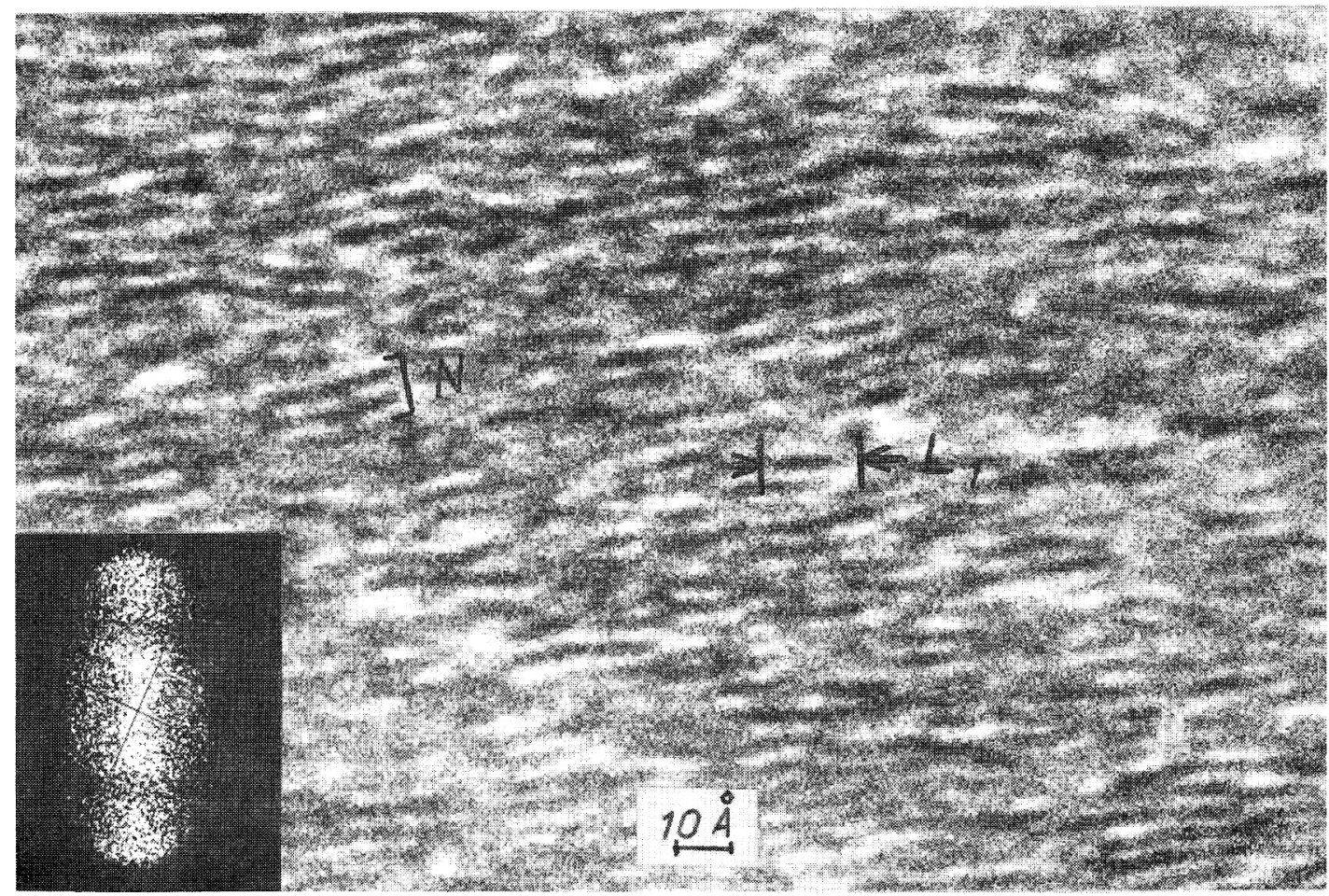

Fig. 6 Soft carbon (HTT $1000^{\circ} \mathrm{C}$ ) lattice imaging and optical diffraction pattern $\times 6,000,000$.

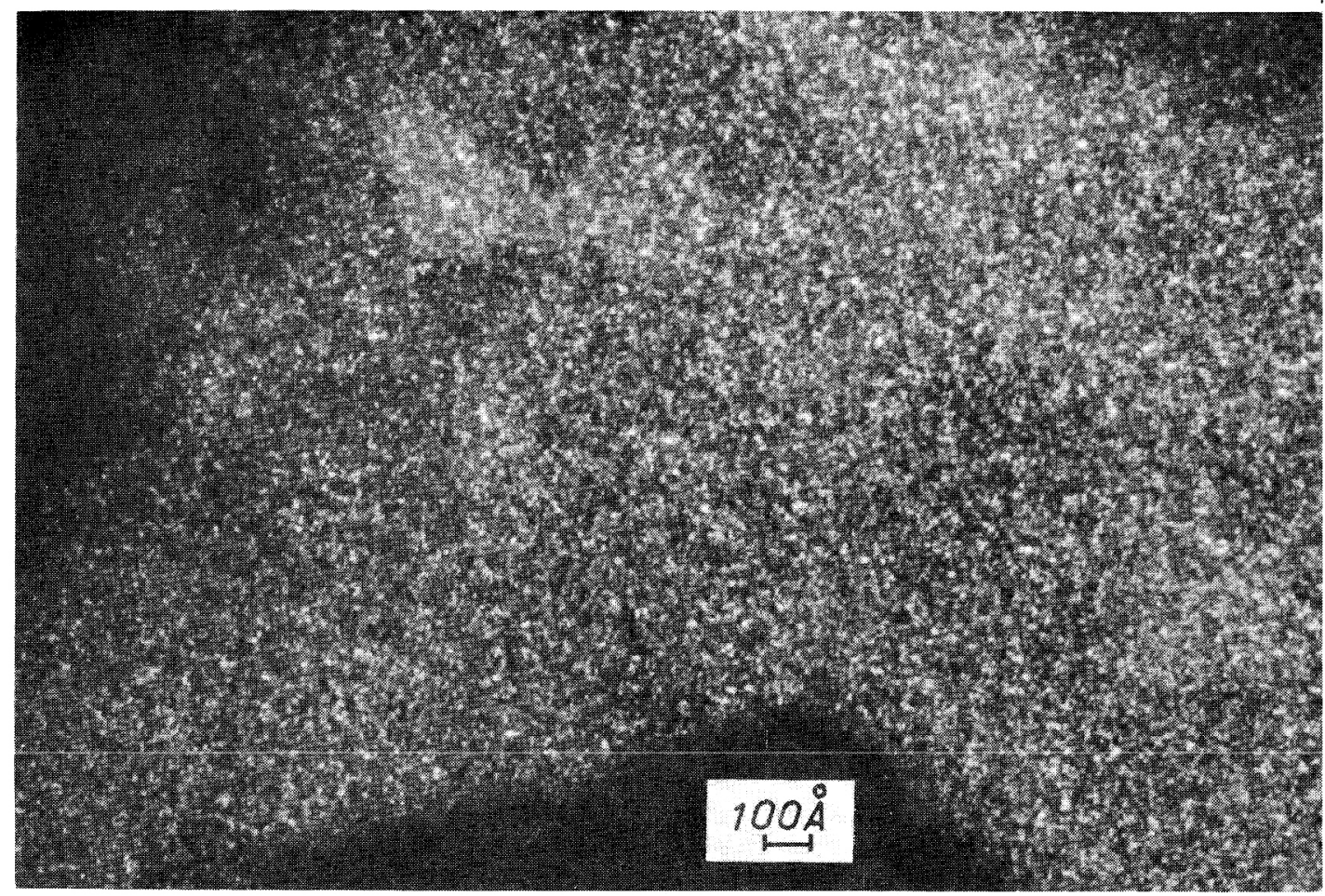

Fig. 7 Soft carbon (HTT $\left.1000{ }^{\circ} \mathrm{C}\right),(11)$ dark-field micrograph $\times 500,000$. 
per stack is generally 2 , and very rarely 3 . All the stacks show a very strong parallel preferred orientation in regions larger than $1 \mu \mathrm{m}^{2}$. By analogy with graphite, we may define for each stack of carbon layers previously described, a $\vec{c}$ axis perpendicular to the layer plane and an $\vec{a}$ axis which is perpendicular to the $C$ - $C$ bond and is contained in the layer plane. From lattice imaging, we may deduce that there is a very strong parallel orientation of the $\vec{c}$ axis in the carbon sample heat-treated at $1000{ }^{\circ} \mathrm{C}$. Because this technique is unable to give any indications either on $\vec{a}$ axis mutual orientation or on the "single layers" (those not grouped in pairs), we have used both (10) and (11) dark-field micrographs in order to obtain the diameter and the shape of the stacks.

Fig. 7 shows the small bright dots which appear in the (11) dark-field micrograph, when some of the (11) reciprocal lines are nearly parallel to the incident beam (aperture centered upon $0,80 \AA^{-1}$ i.e. 1,2 $\AA$ in real space). We must recall here that in turbostratic carbons the (10) and (11) rings are asymmetric bands, presenting their most stepped part toward the origin of the reciprocal space (incident beam) and having their maximum intensity slightly displaced toward the high angles in respect to the true Bragg position. This means that if we move our objective aperture in the Abbe image, from the origin toward the high angles, we first intercept the reciprocal lines forming the Bragg angle with the incident beam (i.e. nearly parallel to the incident beam). To be sure to measure the true diameter of the carbon layer stacks we must use only those diffracted beams corresponding to these reciprocal lines. This may be obtained by considering the dark-field image on the fluorescent screen while moving the aperture toward the high angles in the vicinity of the (11) diffuse band. We stop the displacement of the aperture when the first bright dots appearing reach their maximum intensity (because of the non-negligible width of the aperture, we cannot entirely avoid some slightly oblique stacks which appear in the micrograph as dots less bright than the others). For this sample we have found an average diameter of the small isometric bright regions $\left(L_{a}\right)$ under $10 \AA$. This value is in good agreement with the value of $L_{1}$ deduced from lattice imaging and has the advantage of including the 'single layers'. Fig. 7 also shows that the bright regions are well-separated from each other and are dispersed at random inside the particles. This means that the probability that a given stack gives a diffracted beam through the aperture, is constant throughout the whole particle. Also, the $10 \mu \mathrm{m}$ aperture used for highresolution dark-field micrographs intercepts a $15^{\circ}$ arc of the asymmetric Debye-Scherrer ring. This corresponds to stacks having their $\vec{a}$ axis rotated by less than $\pm 7,5^{\circ}$ around their nearly common $\vec{c}$ axis. From these data we may deduce that there is no preferred parallel orientation of the $\vec{a}$ axis in the sample.

We have tried to apply optical diffraction techniques (OD) to the micrograph of Fig. 6 used as a periodical object, and the result is shown in the insert placed in the proper orientation in respect to the micrograph. In short, the Fraunhofer diffraction pattern of an electron microscope picture is the Fourier transform of the image. In this case, because the image corresponds to interference between the (00.2) beam and the incident beam, the optical diffraction pattern should give the distribution of the interlayer spacings. We have used* an optical diffractometer equipped with $\mathrm{He}-\mathrm{Ne}$ laser for coherent illumination; the electron micrograph is placed slightly beyond the focal plane of a convergent lens and the given pattern is recorded in the back focal plane of the lens (Abbe plane). Theoretically, each individual spot seen in the pattern represents a given $d_{00.2}$ spacing. ${ }^{20)}$ Unfortunately the spots can be due either to interlayer spacings inside a stack or to layers from neighbouring stacks, which are not adjoining layers, so that we have to choose in a somewhat arbitrary manner the maximum and minimum spacings (another artefact may arise from the fact that the exact value of the defocus of the objective lens of the electron microscope is not known). Anyway, the minimum value we have found by this method is $3,3 \AA$ and the maximum one is about 5,5 - $6 \AA$. Because only two arcs are visible on the O.D. pattern (instead of one "Debye-Scherrer ring"), we may deduce that a strong parallel orientation of the fringes is present in the micrograph and we may evaluate the misorientation to $\pm 15^{\circ}$.

From the above data, we may infer a rather important fact which needs to be confirmed: instead of an average value, we have found a large spreading of the $d_{00.2}$ spacings. We thus have measured these spacings, using the dark-field techniques previously described. We have found that the first few bright dots were lit at their maximum intensity for a given position of the incident beam in respect to the aperture corresponding to $7 \AA$ $<d<8 \AA$. When the aperture is displaced toward the smaller $d$ values, these dots disappear while other ones appear. The last ones light up when the aperture corresponds to $3,3 \AA<d<3,5 \AA$. Between these two extreme values we have found a maximum number of bright domains lit at their maximum intensity for $3,6 \AA<d$ $<4,0 \AA$. From these data we may deduce, with more certainty than from optical diffraction, that we have a spreading in the interlayer spacings of the stacks in the

* Acknowledgements should be made to A. Fontanel from the Institut Francais du Petrole who has taken the optical diffraction pattern. 
sample heat-treated at $1000^{\circ} \mathrm{C}$. This spreading extends from $3,3 \AA-3,5 \AA$ to at least $7-8 \AA$. It is striking that even for such a low HTT, some of the layers are grouped in pairs having a $d$ spacing approaching those of graphite, and that this value is the minimum that we can find by means either of optical diffraction or darkfield. This fact seems rather important to us and we take it into special account for all the other samples studied at present.

From $1000{ }^{\circ} \mathrm{C}$ up to $1600^{\circ} \mathrm{C}$ (see Fig. 8) the lattice images show that the number $N$ of layers per stack (full circles) increases linearly until about 10 , whereas the diameter $L_{1}$ (open circles in fig. 8) of the elementary units remains constant. Because of the fast increase of $N$, the thickness of a given stack is large when compared to its diameter (which remains under $10 \AA$ ), so that each stack looks like a small fiber. The lattice imaging also shows that we may follow the same carbon layer through more than one stack (see for instance Fig. 9 corresponding to HTT $1300{ }^{\circ} \mathrm{C}$ ). We thus obtain from the fringes a second parameter $L_{2}$ (full triangles in Fig. 8), higher than $L_{1}$, and which is the diameter of a very defective carbon layer having some kind of puffed-up texture due to the tilt and twist boundaries, which remain unchanged between two neighbouring stacks.

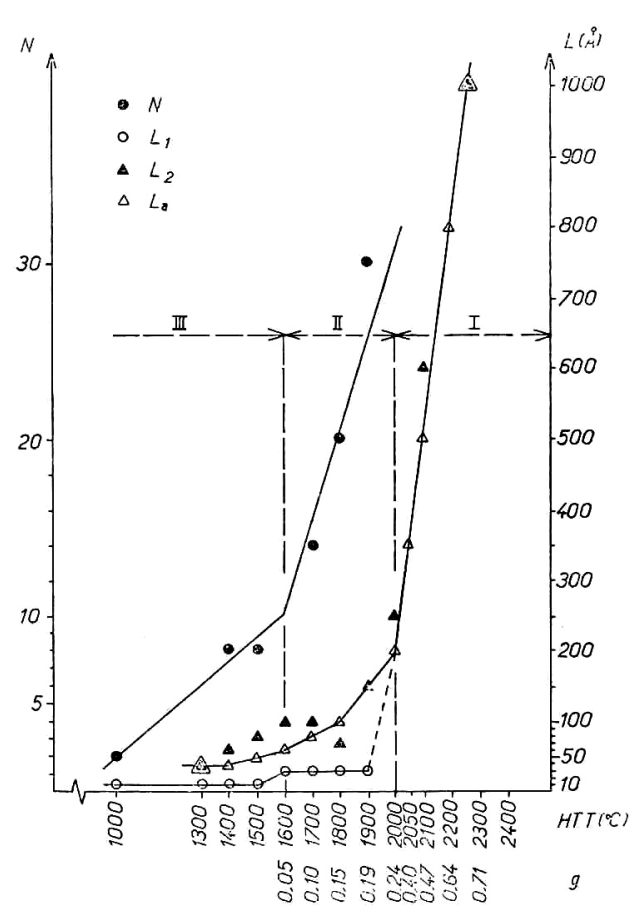

Fig. 8 Variation in size of carbon-layer stacks during heat-treatment.

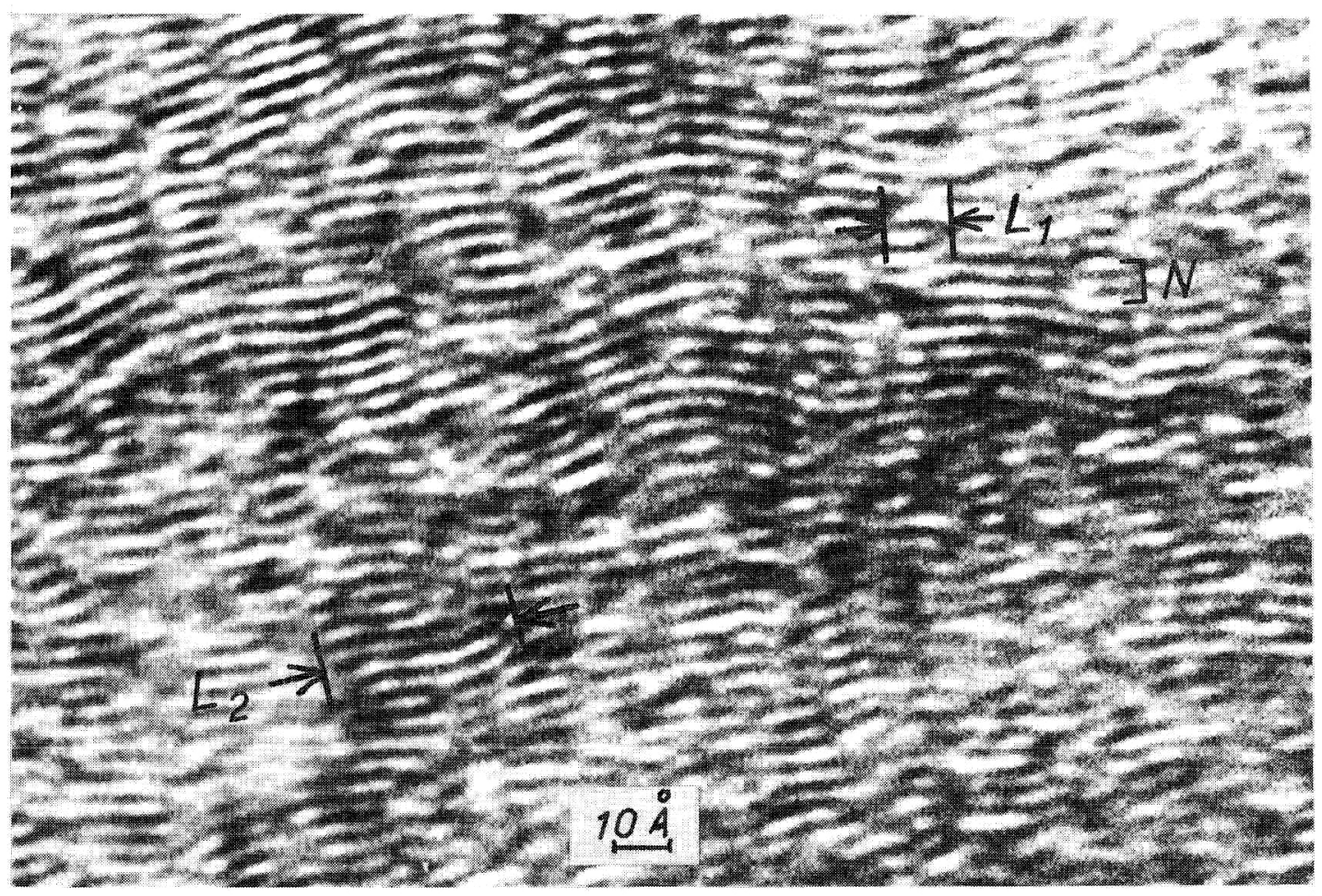

Fig. 9 Soft carbon (HTT $1300^{\circ} \mathrm{C}$ ), lattice imaging $\times 6,000,000$. 


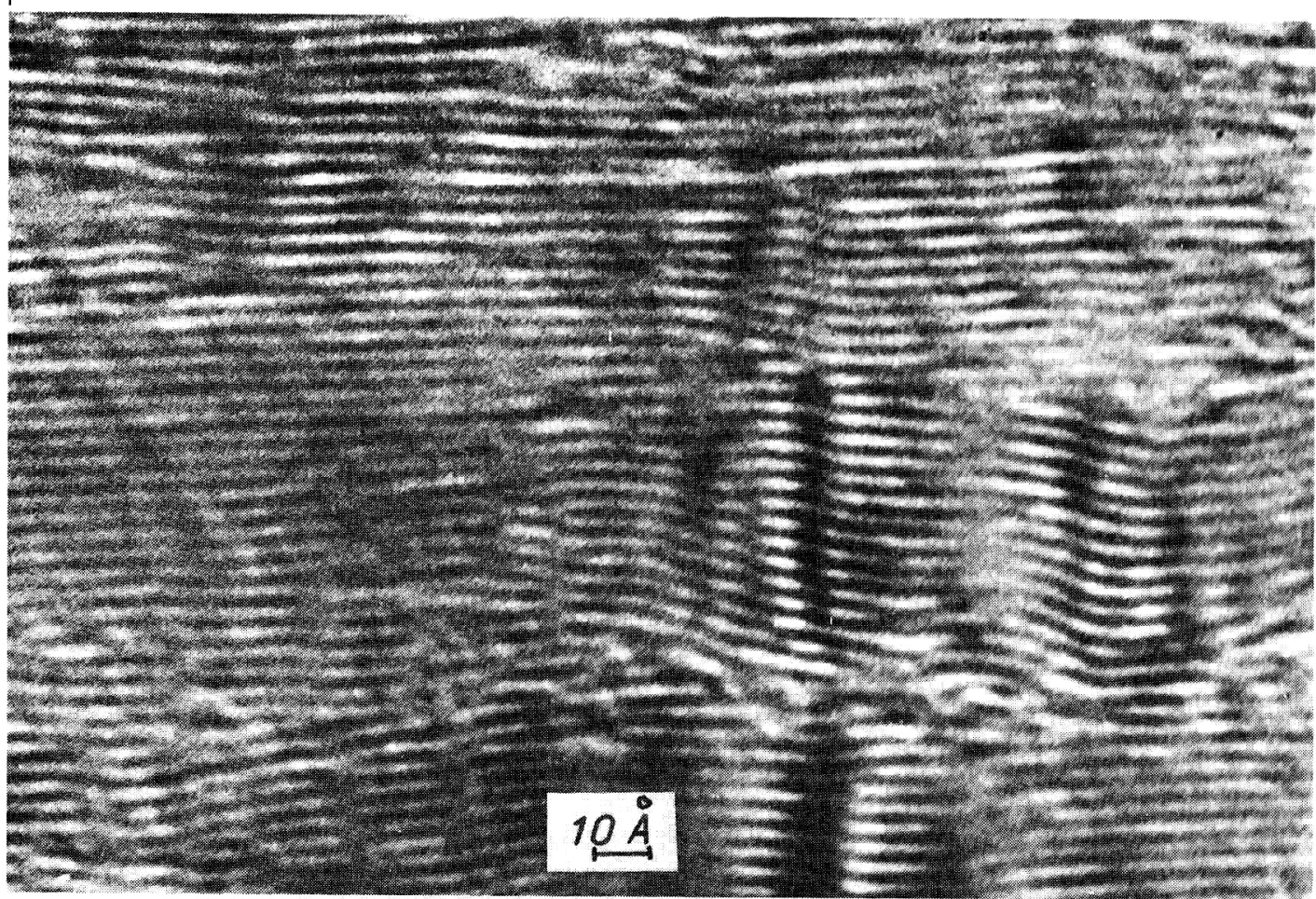

Fig. 10 Soft carbon (HTT $1800^{\circ} \mathrm{C}$ ), lattice imaging $\times 6,000,000$.

If we consider now the (11) dark-field images, we may measure from them the diameter $L_{a}$ (open triangles in Fig. 8) of the bright regions. We have found values superior to $L_{1}$ and in good agreement with $L_{2}$ deduced from lattice imaging. This means that now, two or more neighbouring elementary units give diffracted beams which all go through the aperture at the same time, i.e. their $\vec{a}$ axis are parallel with a maximum misorientation of $\pm 7,5^{\circ}$ around their nearly common $\vec{c}$ axis. And, therefore, they cannot be distinguished from each other in the dark-field micrographs. The increase of $L_{a}$ remains very small in this stage.

\subsubsection{Stage II}

The following stage of transformation corresponds to the increase of the $g$ graphitization factor from 0 to about $0,24\left(1600{ }^{\circ} \mathrm{C}-2000^{\circ} \mathrm{C}\right)$. The diameter $L_{1}$ of the elementary units (flat and defect-free mosaic stacks) suddenly jumps from $10 \AA$ to $30 \AA$ and then remains constant during all this stage. $N$ increases linearly, as before, but with a much higher slope and $L_{2}$, the length of the defective layer, increases faster than in the first stage. Still it keeps its puffed-up texture as shown in Fig. 10 (HTT: $1800^{\circ} \mathrm{C}$ ). The values of $L_{a}$ deduced from (10) or (11) dark-field pictures remain in good agreement with $L_{2}$. This indicates that the growth in diameter of the defective layer is due to the fact that the regions where the $\vec{a}$ axis become nearly parallel, spread out regularly inside the particles.

\subsubsection{Stage I}

The last stage of graphitization corresponds to $g>$ 0,24 (above $2000^{\circ} \mathrm{C}$ ). It is characterized by the apparition in X-ray patterns of the intensity modulations in $(h k)$ bands. The same effect occurs in SAD patterns altogether with the appearance of discrete reflections inside the $(h k)$ rings, which become dotted (indicating a rapid increase of $L_{a}$ ). The fast increase of $L_{a}$ is noticeable in (10) and (11) dark-field images, where large isometric crystallites appear (see Fig. 11) which contain moiré fringes. Unfortunately, just before this stage, the measurements of $N$ from lattice imaging become meaningless, because the number of fringes in lattice images is restricted mainly by the width of the (00.2) extinction contours, due to the increase in crystallinity. The most striking effect which occurs very suddenly at the beginning of this stage (i.e., immediately above $2000{ }^{\circ} \mathrm{C}, g$ $>0,24)$, is the disappearance of the puffed-up aspect. The layers become flat and without any visible defect (see Fig. 12). Also, from $2000^{\circ} \mathrm{C}$ up, no value may be given for $L_{1}$ in the curve of Fig. 8. During this stage, $L_{2}$ as well as $L_{a}$ increase very rapidly. At the same time, the 


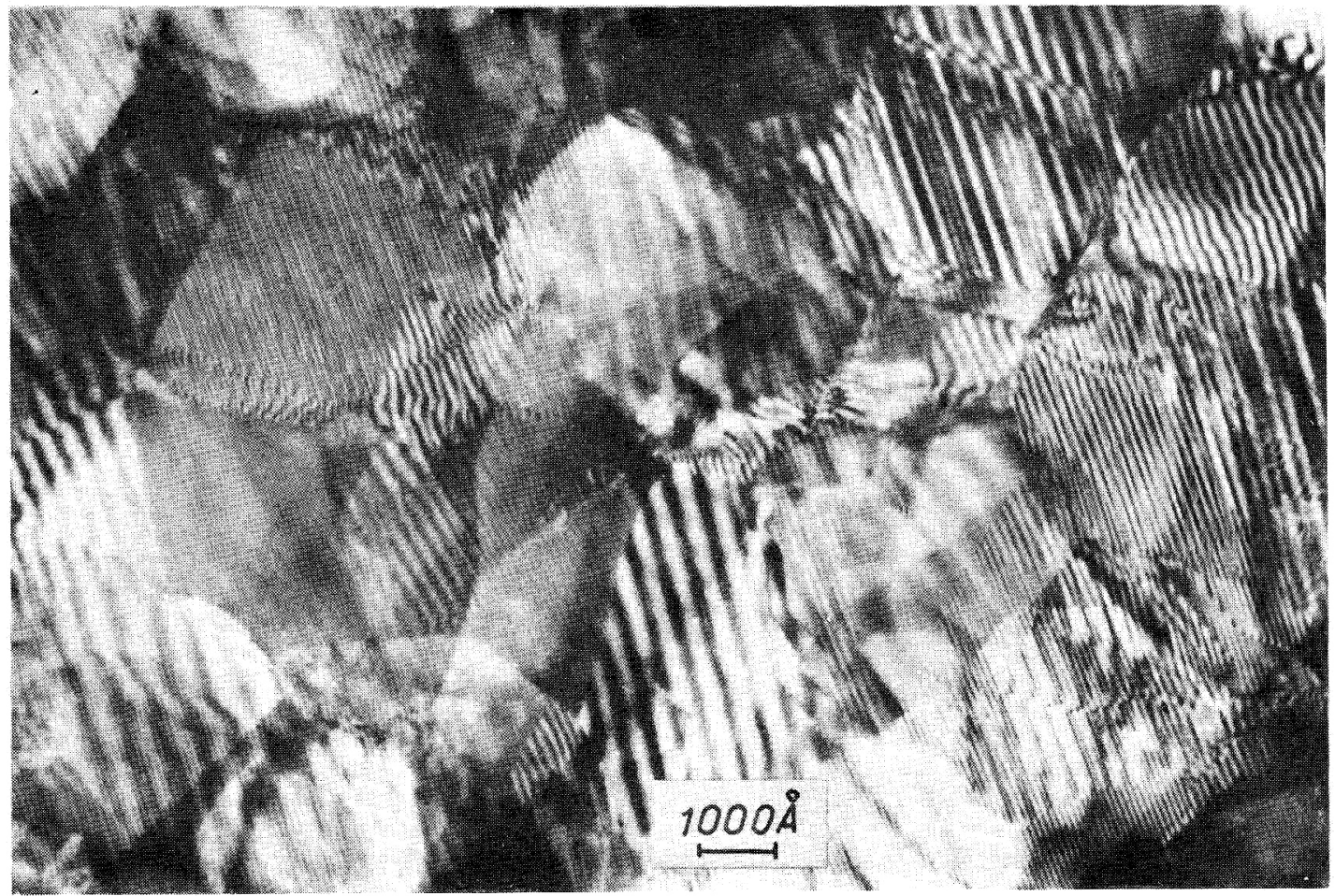

Fig. 11 Soft carbon (HTT $2900{ }^{\circ} \mathrm{C}$ ), (10) dark-field micrograph $\times 80,000$.

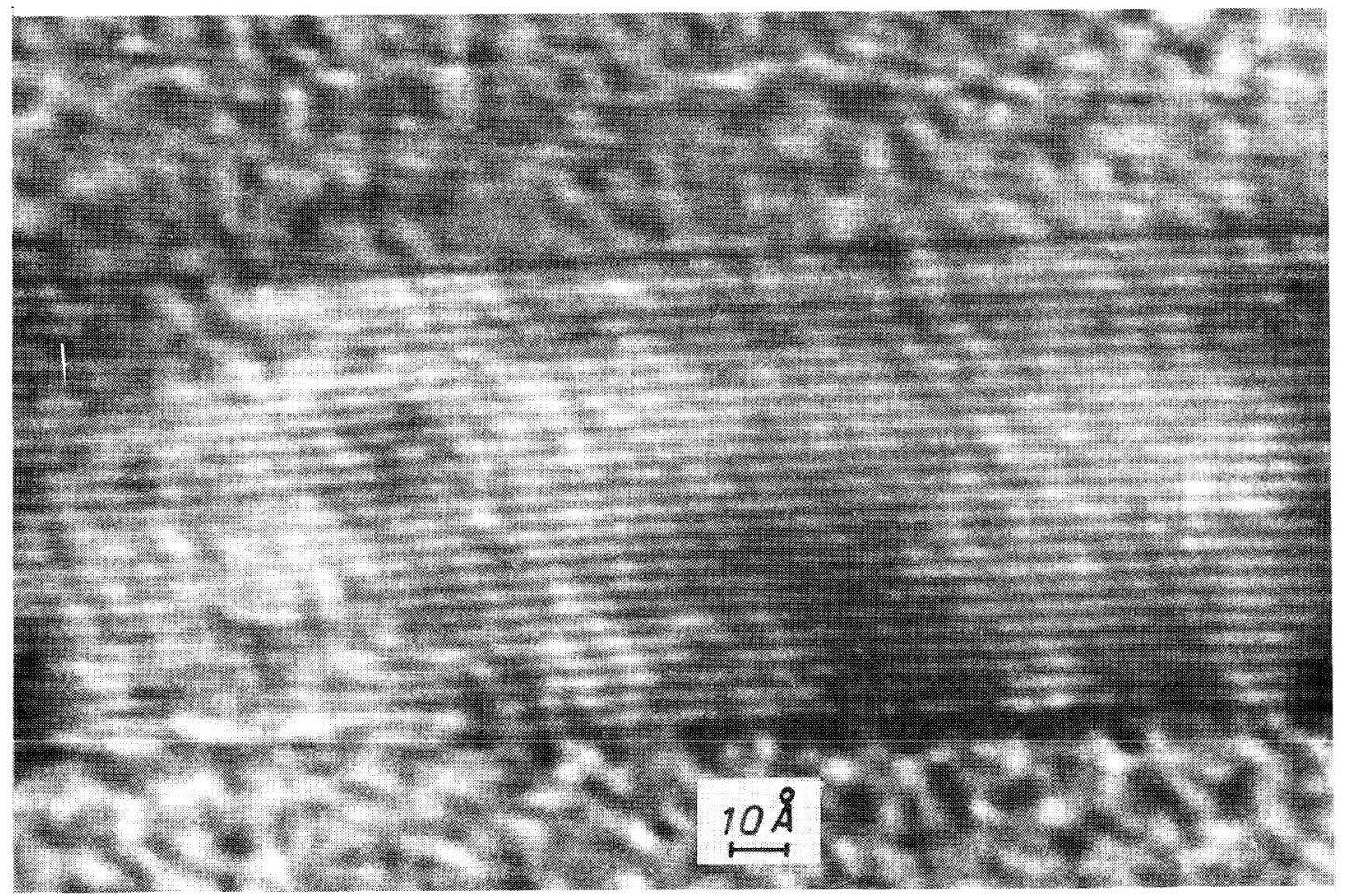

Fig. 12 Soft carbon (HTT 2900 C), lattice imaging $\times 6,000,000$. 
preferred orientation of the carbon layers becomes suddenly perfect. It is only during this stage that the true crystal growth occurs.

To summarize, during stage III the small elementary units (stacks of 2 layers whose diameter is under $10 \AA$ ) are parallel ( $\vec{c}$ axis parallel) but at random in the plane of the preferred orientation ( $\vec{a}$ axis at random). Because of strong parallel orientation of the $\vec{c}$ axis, the increase in thickness of the stacks is favoured. On the contrary, the fact that $\vec{a}$ axis are at random prevents a fast increase in diameter. In stage II the thickness increases faster, more and more $\vec{a}$ axis tend to become nearly parallel, some defects disappear $\left(L_{1}\right.$ jumps from $10 \AA$ to $30 \AA$ ), but many of them remain frozen-in at the boundaries of the initial elementary stacks, so that tilt and twist boundaries remain inside the growing layer which is very defective. In stage $I$, the defects at the boundaries are suddenly wiped out, tilt and twist boundaries suddenly disappear and real crystal growth occurs.

These results suggest that graphitization proceeds through progressive coalescence of very small elementary units. These units, less than $10 \AA$ in diameter, are formed by two aromatic layers, which show a very large spreading in their interlayer spacing from 7-8 to a minimum value approaching those of graphite (about 3,3-3,5 $\AA$ ). We must take this fact into account because this spreading must be sufficiently reduced during carbonization process (below $1600^{\circ} \mathrm{C}$ ) to allow graphitization process to begin. On the other hand, the graphitization process is greatly helped by the occurrence, during the carbonization stage, of a strong preferred orientation of these small units whose $\vec{c}$ axis are nearly parallel, inside very large regions of the bulk $\left(>1 \mu \mathrm{m}^{2}\right)$. Nevertheless, the fact that, at first, the $\vec{a}$ axis of these elementary units are at random, causes (during the subsequent growth) the formation of very defective layers containing many frozen-in defects which are responsible for the development of strong internal stresses. Only when some atomic mobility occurs, (above $2000^{\circ} \mathrm{C}$ ) may those stresses be relieved very suddenly by the wiping out of the defects. Only at this moment do flat unconstrained layers appear, preceding the true crystal growth. These results are in fairly good agreement with the data obtained by other workers ${ }^{21,22,6)}$ and should help to obtain a coherent view of graphitization process.

\subsection{Hard Carbons (heat-treated above $2000^{\circ} \mathrm{C}$ )}

In a previous work $23,24,25,26)$ we have studied numerous hard carbons, including carbons from very pure saccharose, cellulose, polyvinylidene chloride, furfurylic acid, polyurethan and glassy carbon. All samples have been heat-treated from $2000^{\circ} \mathrm{C}$ to $3000^{\circ} \mathrm{C}$ and at the present time the work (still unpublished) is still running upon samples heat-treated below $2000{ }^{\circ} \mathrm{C}$. All the samples studied have given us similar results. Consequently we will consider here that our data are representative for hard carbons.

\subsubsection{Matrix}

The particles obtained by gentle grinding have no special shape and always give SAD patterns containing both intense (00.2) rings and diffuse asymmetric $(h k)$ bands. This confirms the statistical randomness of the carbon layers as seen by X-rays (loc. cit. 3,4). The porous structure of these carbons is directly visible in bright-field micrographs as shown in Fig. 13 and we have assumed that the pores appear as clearer areas than the walls which appear themselves as a net of very sinuous dark lines*. We have noticed that the pore size increases from $2000{ }^{\circ} \mathrm{C}$ to $3000^{\circ} \mathrm{C}$ and we have evaluated this size to be in the vicinity of $50 \AA$ for samples heattreated at $2700{ }^{\circ} \mathrm{C}$. This value may be compared with $\mathrm{X}$-ray small-angle scattering data. ${ }^{27}$ ) Whatever the heattreatment temperature, the (00.2) dark-field pictures show that the carbon layers are parallel to the pore walls. These data are confirmed by those of lattice imaging technique. The picture in Fig. 14 which corresponds to $\mathrm{HTT} 2500^{\circ} \mathrm{C}$ shows that the carbon layers form the walls of polyhedral pores whose average size is about 30-60 $\AA$. In a wall the number of layers per stack is often 7 , more rarely 5 to 10 and the length of the fringes gives a value of about $70 \AA$ for the layer diameter (fig. 15). Because we cannot deduce the real shape of the stacks from lattice imaging, we have used (10) and (11) dark-field images. Fig. 14 shows isometric bright regions of about $100 \AA$ in diameter, which clearly proves that elementary domains of hard carbons are not ribbonlike. ${ }^{28)}$

\subsubsection{Graphitic phases}

As Franklin has shown (loc. cit. 3, 4) hard carbons are not graphitizable and no graphitization degree may be measured from them because they remain turbostratic. However, X-ray diffraction data indicate that above $2200{ }^{\circ} \mathrm{C}$, some samples suddenly become a mixture of three phases whose total amount remains unchanged up to $3000{ }^{\circ} \mathrm{C}$. The two new phases are a graphite phase and a graphitizable one which appear at the same time.

\footnotetext{
* The contrast of the dark lines is enhanced above $2000{ }^{\circ} \mathrm{C}$. This is due to the fact that the aperture placed in the Abbe plane withdraws the difUnder $2000{ }^{\circ} \mathrm{C}$, for a given sample, most of the $(00.2)$ spacings are larger than this value and thus some $(00.2)$ beams may reach the optical image, giving a poor contrast between pores and walls. Above $2000{ }^{\circ} \mathrm{C}$ the $(00.2)$ spacings decrease enough to cause the $(00.2)$ beams to be stopped by the aperture, thus enhancing the contrast.
} 


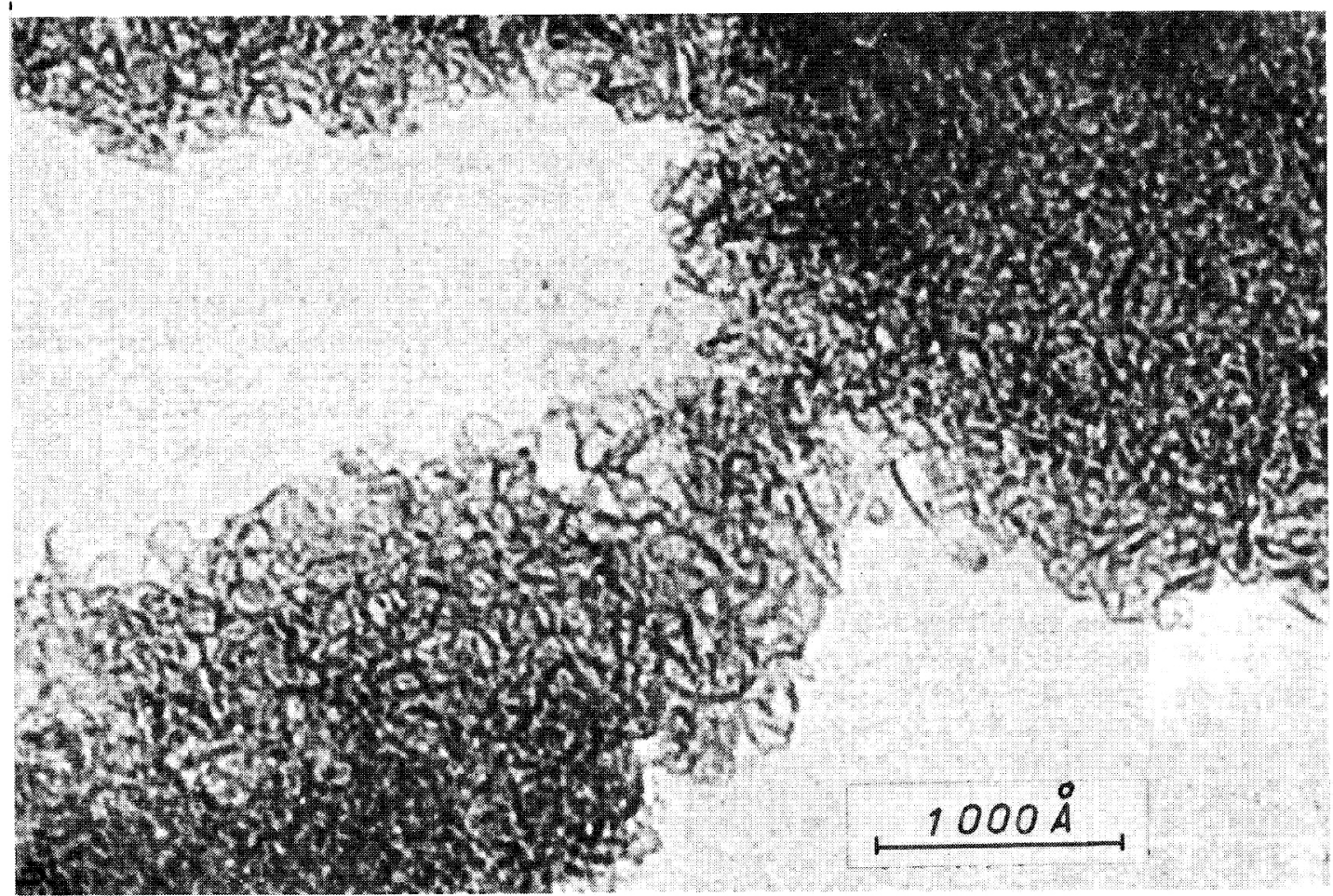

Fig. 13 Hard carbon $\left(\right.$ HTT $2700{ }^{\circ} \mathrm{C}$ ), bright-field $\times 270,000$.

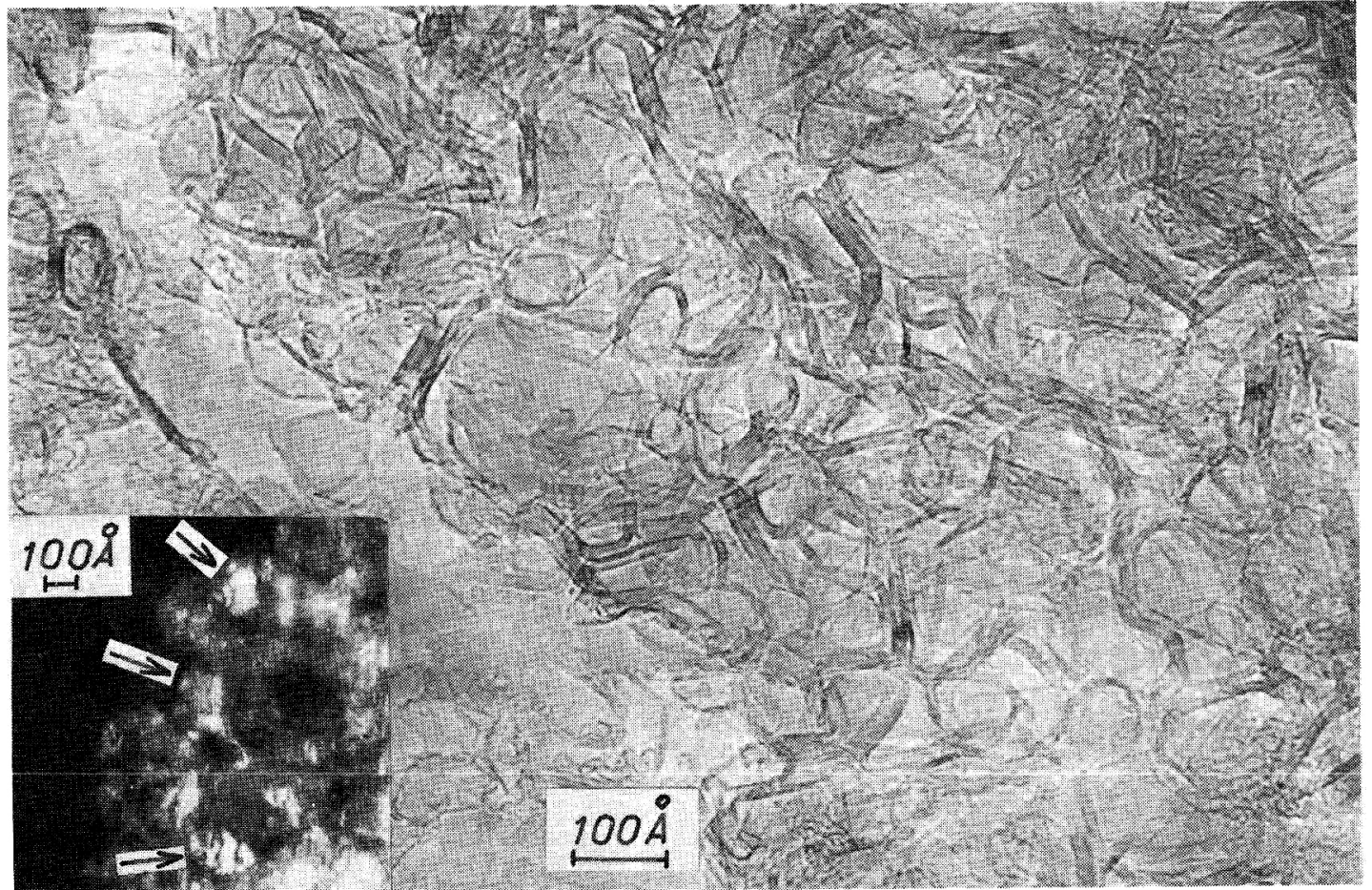

Fig. 14 Hard carbon, comparison of bright-field micrograph (HTT $2500^{\circ} \mathrm{C}$ ) $\times 1,000,000$ and $(11)$ dark-field $\left(\right.$ HTT $\left.2700{ }^{\circ} \mathrm{C}\right) \times 300,000$. 


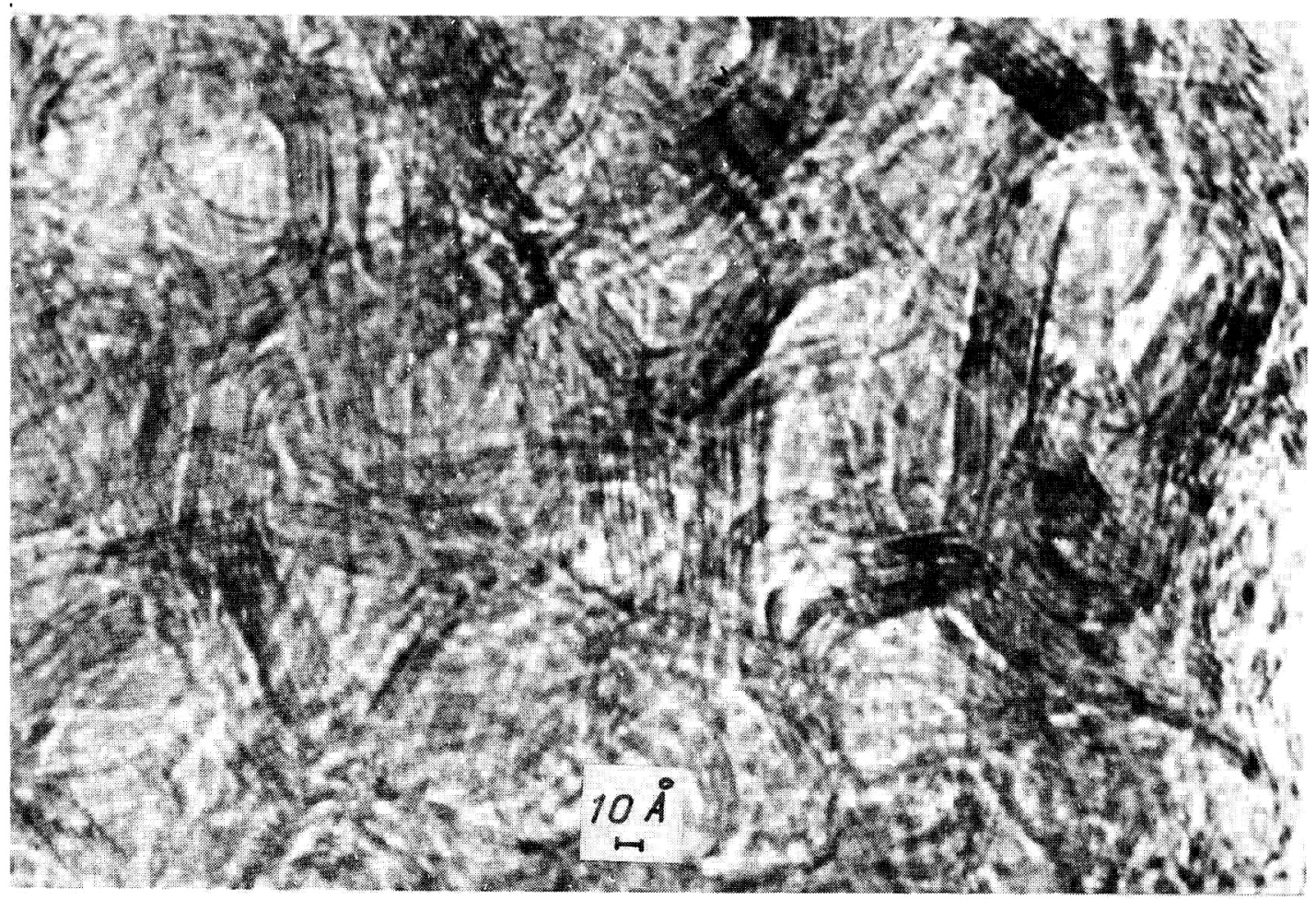

Fig. 15 Hard carbon (HTT $2800^{\circ} \mathrm{C}$ ), lattice imaging $\times 3,000,000$.

We have shown (loc. cit. 23 to 26) that this was a general phenomenon, encountered in all kinds of hard carbons, and that these additional graphite phases were gathered in a shell surrounding the carbon grains. This shell is visible by means of the optical microscope, since it is anisotropic under polarized light.

The morphology of the graphitic phases is very typical and quite different from the matrix. The graphite particles are lamellar and the graphitizable ones are, in fact, conical spirals ${ }^{29)}$ of turbostratic carbon layers grown in a nearly spherical shape. When HTT increases from $2200{ }^{\circ} \mathrm{C}$ to $3000{ }^{\circ} \mathrm{C}$ the graphitizable carbon particles suddenly transform one by one, in an increasing number, into graphite. At $3000{ }^{\circ} \mathrm{C}$ we obtain a mixture of only two phases - non graphitized matrix and graphite.

We have also shown that when using a very compact sample of hard carbon (i.e. plates of glassy carbon instead of powder), neither anisotropy under polarized light, nor particles of graphitic phases occur above $2200^{\circ} \mathrm{C}$. We have only seen the turbostratic matrix. These results may explain those obtained by Kawamura and Tzusuku, ${ }^{30)}$ who found that when glassy carbon becomes more porous, its "degree of graphitization" (as evaluated by magnetoresistance and resistivity measurements) increases.
These data suggest that Franklin's model may be used for hard carbons with only slight modifications concerning the texture of the matrix and provided that we remember that two kinds of graphitic phases occur instead of either one or two. In fact, the porous material, when heat-treated above $2000^{\circ} \mathrm{C}$, contains isometric elementary domains whose diameter is about $70-100 \AA$ and which form the walls of polyhedral isometric pores. We consider a foam as a satisfactory model of such a texture. This texture is responsible for the occurrence, during heat-treatment, of very strong internal stresses, which cannot be relieved because of the strong bonding between carbon layers where pore walls are merging. These stresses are only relieved, above $2000{ }^{\circ} \mathrm{C}$, when atomic mobility occurs, producing the "catastrophic" growth of the two graphitic phases outside the external surface of the particles (anisotropic shell). Because of the relief of the stresses, the energy added by subsequent heat-treatment is not sufficient to improve the mutual orientation of the carbon layer stacks, so that they remain at random in the matrix. Because of their size (about $100 \AA$ ), the flat, unconstrained parallel layers (compare fig. 14 and 15 to fig. 11 and 12) remain grouped in turbostratic stacks. Graphitization is hindered here by purely geometrical effects. This behaviour is probably similar to that of acetylen blacks. In spite of their small size, those blacks are able to polygonize but remain turbostratic, because the flat layers grouped in stacks are smaller than 
$200 \AA^{31}$ ). (end of part I)

\section{BIBLIOGRAPHY}

1. J. Millet, J.E. Millet and A. Vivares, J. Chim. Phys., 60, 553 (1963).

2. K. Ouchi, paper, 117-1-29-A 01

3. R. Franklin, Proceed. Roy. Soc., 209, 196 (1951).

4. R. Franklin, Acta Cryst., 3, 107 (1950).

5. M. Shiraishi and K. Kobayashi, Bull. Chem. Soc. Japan, 46, 2575 (1973).

6. D.B. Fischbach, Chemistry and Physics of Carbon, 7, 1 (1971), Dekker N.Y. Ed.

7. J. Mering and A. Oberlin, The Electron Optical Investigations of Clays, 197 (1971), Mineralogical Soc. London ed.

8. J. Maire, These (1967), Schiffer Paris ed.

9. J. Mering and J. Maire, J. Chim. Phys., 57, 801 (1960) suppl.

10. C. Schiller and J. Mering, Carbon 5, 385 (1967).

11. N. Ueda, T. Kobayashi, E. Suito, Y. Harada and M. Watanabe, J. Appl. Phys., 43, 5181 (1972).

12. P.B. Hirsch, A. Howie, R.B. Nicholson, D.W. Pashley and M.J. Whelan, Electron Microscopy of Thin Crystals, 353 (1971), Butterworth, N.Y. ed.

13. T. Komoda, Optik, 21, 93 (1964).

14. A. Howie, O.L. Krivanek and M.L. Rudee, Phil. Mag., 27, 235 (1973).

15. A. Oberlin and G. Terriere, J. Microscopie, 14, 1 (1972)

16. A. Oberilin, G. Terriere and J.L. Boulmier, Philips
Bulletin (1974) (in preparation).

17. A. Oberlin and G. Terriere, 11th Biennal Conf. Carbon, Gatlinburg 24 (1973).

18. A. Oberlin and G. Terriere, J. Microscopie, 18, 247 (1973).

19. H. Akamatu and H. Kuroda, 4th Conf. Carbon, 355 (1960) Pergamon ed.

20. L.L. Ban, P.C. Vegvari and W.M. Hess, Norelco Reporter, 20, 1 (1973).

21. H. Takahashi, H. Kuroda and H. Akamatu, Carbon, 2, 432 (1965).

22. M. Inagaki, Y. Murase, H. Saito and T. Noda, Bull. Chem. Soc. Japan, 43, 942 (1970).

23. A. Oberlin and F. Rousseaux, J. Appl. Cryst., 1, 218 (1958).

24. A. Oberlin and F. Rousseaux, J. Appl. Cryst., 3, 105 (1970).

25. A. Oberlin, F. Rousseaux and J.P. Rouchy, Rev. Int. Hautes Temper. et Refract., 7, 128 (1970).

26. ibid., Tanso 75, 141 (1973).

27. R. Perret and W. Ruland, J. Appl. Cryst., 1, 308 (1968).

28. G.M. Jenkins and K. Kawamura, Nature, 231, 175 (1971).

29. T. Tsuzuku, Proc. 3rd Conf. Carbon, 433 (1957), Pergamon ed.

30. K. Kawamura and T. Tsuzuku, Carbon, in press (1974).

31. G. Terriere, M. du Besset, A. Oberlin and A. Pacault, Carbon, 7, 385 (1969). 